\title{
Susanne Bender: Grundlagen der Tanztherapie. Geschichte, Menschenbild, Methoden
}

Psychosozial-Verlag, 2020, Gießen, 330 Seiten, 34,90€

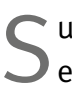
usanne Bender ist Tanztherapeutin, anerkannte Ausbilderin, Lehrtherapeutin und Supervisorin beim BTD, Sonderpädagogin und Heilpraktikerin für Psychotherapie. Mit „Grundlagen der Tanztherapie“ veröffentlichte sie ihr inzwischen drittes Buch. Im ersten Teil beschreibt sie sehr anschaulich die Entstehungsgeschichte der Tanztherapie. In lebendiger Weise wird deutlich, aus welchen Quellen die Tanztherapie gespeist wird, wie und weshalb die Mündung in den Beruf hinein entstanden ist und wie die Tanztherapie heute in Deutschland und auch in Europa aufgestellt ist.

Anhand der philosophischen und zeitgeschichtlichen Auseinandersetzungen mit dem Menschenbild für die Tanztherapie beschreibt die Autorin detailliert, was z. B. unter dem tanztherapeutischen Begriff „Körper-Geist-SeeleEinheit" gemeint ist und welches Menschenbild die TanztherapeutInnen in ihren Handlungen leiten kann. Sie schreibt am Ende dieses Kapitels (S.127): „In der Tanztherapie geht es darum, den Menschen an die Tiefendimensionen seines Erlebens und somit an die Ganzheitlichkeit seines Seins heranzuführen.“

Grundlegende Erkenntnisse, auch weiterer erfahrener TanztherapeutInnen, bilden im Mittelteil des Buches ein Nachschlagewerk für alle relevanten Themen, die im Praxisalltag, im stationären wie ambulanten Setting, zum Tragen kommen. Zahlreiche kreative Methoden, Techniken und Interventionen mit erklärenden Fallbeispielen aus der Praxis erläutern das Handwerkszeug von TanztherapeutInnen und schlieBen sich daran an.
Gerade in der Auseinandersetzung mit dem Menschenbild in der Tanztherapie regt die Autorin eine interessante Diskussion unter TanztherapeutInnen an; ihre weitreichenden Recherchen und Begriffsklärungen tragen zum Verständnis und Überdenken des Alltagsgebrauchs von Sprache in diesem Kontext bei.

Das vorliegende Buch beleuchtet die vielseitigen Möglichkeiten der Therapiemethode, und gleichzeitig wird dem Leser das große Fundament, auf dem die Methode ruht, deutlich. Beim Lesen wird spürbar, dass sich die Autorin ganz bewusst für die in der Praxis erprobten, relevanten Themen der Tanztherapie einsetzt und mit großem Engagement ihr reiches Wissen weitergibt. Ihre Liebe zum Detail macht deutlich, dass sie sich in der Tiefe in den von ihr beschriebenen Themen der Tanztherapie zuhause fühlt und auch die in der Methode ungeübten LeserInnen mitnehmen kann. Ein Überblick über die Therapiemethode wird durch zahlreiche Zitate aus den angrenzenden Kontexten umrahmt.

Mit diesem Buch legt sie ein Grundlagenwissen vor, das sowohl Studierenden als auch den in der Praxis tätigen Therapeutlnnen zu empfehlen ist.

\section{Martina Maria Meshesha}

DOI 10.2378/ ktb2021.arto6d 